\title{
The gamma-ray signal from type IIP core-collapse supernovae
}

\section{Pierre Cristofari, ${ }^{a, *}$ Matthieu Renaud, ${ }^{b}$ Alexandre Marcowith, ${ }^{b}$ Vincent Tatischeff ${ }^{c}$ and Vikram Dwarkadas ${ }^{d}$}

${ }^{a}$ Observatoire de Paris, 5 Place Jules Jansen, 92195, Meudon, France

${ }^{b}$ Laboratoire Univers et Particules de Montpellier (LUPM)

Université de Montpellier, CNRS/IN2P3, CC72, place Eugène Bataillon

F-34095 Montpellier Cedex 5, France

${ }^{c}$ Université Paris-Saclay, CNRS/IN2P3

IJCLab, 91405 Orsay, France

${ }^{d}$ Department of Astronomy and Astrophysics

University of Chicago, 5640 S Ellis Ave, Chicago, IL 60637, USA

E-mail: pierre.cristofari@obspm. fr

The shock wave resulting from the core-collapse of a massive star can accelerate particles up to $\mathrm{PeV}$ energies in the first few days to weeks after the explosion. This can lead to the production of a potentially detectable gamma-ray signal. The gamma-ray flux however is strongly affected by the two photon-annihilation process, where gamma-ray photons interact with photons from the SN photosphere. This process hampers the detection of gamma-rays from core-collapse supernovae at very high energies. In order to probe the detectability of the gamma-rays with current and upcoming gamma-ray facilities, we estimate the gamma-ray flux from typical type IIP core collapse supernovae (CCSNe). These are the most common type of supernovae, and are presumed to arise from red supergiant progenitors. We include a detailed time-dependent calculation of two-photon absorption. Our results will be very useful in creating a strategic observing program to detect CCSNe with the next generation gamma-ray observatory, such as the Cherenkov Telescope Array (CTA).

$37^{\text {th }}$ International Cosmic Ray Conference (ICRC 2021)

July 12th-23rd, 2021

Online - Berlin, Germany

\footnotetext{
*Presenter
} 


\section{Introduction}

In the first days and weeks after the explosion of core-collapse supernovae (CCSNe), it has been shown that efficient particle acceleration can energized hadrons up to the $\gtrsim \mathrm{PeV}$ range through the first-order Fermi mechanism, diffusive shock acceleration (DSA). The accelerated hadrons can in turn interact with the dense circum-stellar environment and produce gamma rays, through pion creation-decay [1-15]. A naive estimate of the gamma-ray signal thus created shows that a detection of extraGalactic CCSNe located within a few $\lesssim \mathrm{Mpc}$, by next-generation instruments operating in the $\sim 10 \mathrm{GeV}$ to $\sim 100 \mathrm{TeV}$ range is a priori conceivable. However, the gamma-ray signal from hadronic interactions of accelerated particles with their surrounding can be degraded by interactions with low energy photons from the SN photosphere, leading to the creation of electron-positron pairs. This two-photon process has been shown to be very important in the first days to weeks after the explosion of a CCSN, reducing the gamma-ray signal by several orders of magnitude, and a careful calculation of the level of attenuation is essential in order to investigate the chances of detection in the gamma-ray range with future instruments. The inclusion of time effects, including for instance the time evolution of the photospheric radius and temperature, of the shock radius and the time of flight of photons before interaction is thus crucial in this problem. The importance of these time effects has been shown in the case of the very well studied SN1993J [16, 17]. The case of SN1993J was especially instructive, since it likely corresponded to a type IIb $\mathrm{SN}$, and thus a rather energetic event, and thus would have been a good candidate for the detection with gamma-ray instruments. However, such type of CCSNe is expected to be infrequent, and the bulk of CCSNe might be less luminous in the gamma-ray domain. Using a simple analytical description of the photosphere and shock evolution, we investigate the gamma-ray signal from typical core-collapse type IIP SNe, taking into account the degradation due to the two-photon process. We find that the gamma-ray signal of only nearby objects, typically located within $\lesssim 1 \mathrm{Mpc}$ is somewhat at the level of the typical sensitiviy of next-generation Cherenkov instruments in the $\sim 10 \mathrm{GeV}$, and that above the $\gtrsim 100 \mathrm{GeV}$ range, the chances of detection are reduced [18].

\section{The gamma-ray signal from type IIP CCSNe}

As mentioned before, the detectability in the gamma-ray domain is mainly limited by the two-photon process, that we intend to take into account. We rely on an estimate of the gamma-ray signal from the shell around the SN shock wave expanding through the dense wind of its progenitor red super giant (RSG) star [1, 19]:

$$
\begin{array}{r}
\frac{\mathrm{d} N}{\mathrm{~d} E} \approx 4.8 \times 10^{-14}\left(\frac{\xi_{\mathrm{CR}}}{0.1}\right)\left(\frac{\dot{M}_{\mathrm{w}}}{10^{-6} \mathrm{M}_{\odot} / \mathrm{yr}}\right)^{2}\left(\frac{v_{\mathrm{w}}}{10^{6} \mathrm{~cm} / \mathrm{s}}\right)^{-2} \\
\times\left(\frac{D}{\mathrm{Mpc}}\right)^{-2}\left(\frac{v_{\mathrm{sh}}(t)}{10^{9} \mathrm{~cm} / \mathrm{s}}\right)^{2}\left(\frac{R_{\mathrm{sh}}(t)}{10^{14} \mathrm{~cm}}\right)^{-1}\left(\frac{E}{1 \mathrm{TeV}}\right)^{-2} \mathrm{TeV}^{-1} \mathrm{~cm}^{-2} \mathrm{~s}^{-1},
\end{array}
$$

where $\xi_{\mathrm{CR}}=P_{\mathrm{CR}} / \rho_{\mathrm{w}} v_{\mathrm{sh}}^{2}$ is the normalised CR pressure, $\dot{M}_{\mathrm{w}}$ the mass-loss rate of the progenitor wind, $v_{\mathrm{w}}$ the wind speed of the progenitor RSG, $D$ the distance, $v_{\mathrm{sh}}$ the shock speed, and $R_{\mathrm{sh}}$ the shock radius. In the first days/weeks after the explosion, the evolution of the photospheric radius 
and photospheric temperature can be described analytically [20]:

$$
\begin{array}{r}
r_{\mathrm{ph}}(t)=2.9 \times 10^{14}\left(\frac{f}{0.1}\right)^{-0.062}\left(\frac{E_{\mathrm{SN}}}{10^{51} \mathrm{erg}}\right)^{0.41}\left(\frac{\kappa}{0.34}\right)^{0.093} \\
\times\left(\frac{M_{\mathrm{ej}}}{M_{\odot}}\right)^{-0.28}\left(\frac{t}{\text { days }}\right)^{0.81} \mathrm{~cm} \\
T_{\mathrm{ph}}(t)=1.7\left(\frac{f}{0.1}\right)^{-0.037}\left(\frac{E_{\mathrm{SN}}}{10^{51} \mathrm{erg}}\right)^{0.027}\left(\frac{R_{\star}}{10^{13} \mathrm{~cm}}\right)^{1 / 4}\left(\frac{M_{\mathrm{ej}}}{M_{\odot}}\right)^{-0.054} \\
\times\left(\frac{\kappa}{0.34 \mathrm{~cm}^{2} / \mathrm{g}}\right)^{-0.28}\left(\frac{t}{\text { days }}\right)^{-0.45} \mathrm{eV}
\end{array}
$$

where $f$ is a numerical parameter found for typical RSG in the range [0.079,0.13], $M_{\mathrm{ej}}$ is the ejecta mass, $E_{\mathrm{SN}}$ the total explosion energy, $R_{\star}$ the radius of the progenitor star, and $\kappa$ the opacity, assumed to be time-and-space-independent.

The evolution of the SN shock can be described with self-similar solution [21], and reads,for a slope of the density profile of the envelope of the exploding star $n=10$ :

$$
\begin{aligned}
R_{\mathrm{Sh}}(t) \approx 4.710^{14}\left(\frac{E_{\mathrm{SN}}}{10^{51} \mathrm{erg}}\right)^{0.44}\left(\frac{\dot{M}_{\mathrm{w}}}{10^{-6} \mathrm{M}_{\odot} / \mathrm{yr}}\right)^{-0.17}\left(\frac{M_{\mathrm{ej}}}{\mathrm{M}_{\odot}}\right)^{-0.31} & \times\left(\frac{t}{\text { days }}\right)^{0.875} \mathrm{~cm}
\end{aligned}
$$

These quantities are then used to derive the absorption through pair production including time effects, as described in [17], but in the case of type IIP CCSNe.

\section{Results}

The physical quantities used to describe the type IIP SNe $\left(\xi_{C R}, M_{\mathrm{ej}}, \dot{M}, R_{\star}\right.$ and $E_{\mathrm{SN}}$ can change the level of the gamma-ray emission and the level of the attenuation, but in all case, exploring the parameter space, we find that the gamma-ray signal from a type IIP SN located at $1 \mathrm{Mpc}$ is at most at the level of the expected sensitivity of next-generation instruments. This is illustrated in Fig. ??. Moreover, we find that the $\sim 10 \mathrm{GeV}$ domain will contribute to a potential detection, and that in turn, the detection of gamma-ray above $\gtrsim 100 \mathrm{GeV}$ in the first month will be very challenging, if possible at all. This strongly limits the possibility of detection of typical type IIP SNe located further than $\gtrsim 1 \mathrm{Mpc}$ and the possibility of probing particle acceleration to the highest energies using gamma rays. Type IIP SNe represent the most important fraction of CCSNe [22], but are likely to be the worst candidates in terms of detection in the gamma-ray domain, even though other effects, such as enhanced mass loss prior to the explosion might help increase the chances of detection. However, other, more powerful CCSNe (such as type IIb or IIn) might be more promising and could expand the horizon of detectability, thus increasing the chances of detection of gamma rays from $\mathrm{SNe}$ in the first days and weeks after the explosion, especially increasing the chances to probe the acceleration up to the $\mathrm{PeV}$ range at $\mathrm{SNe}$ [23]. 

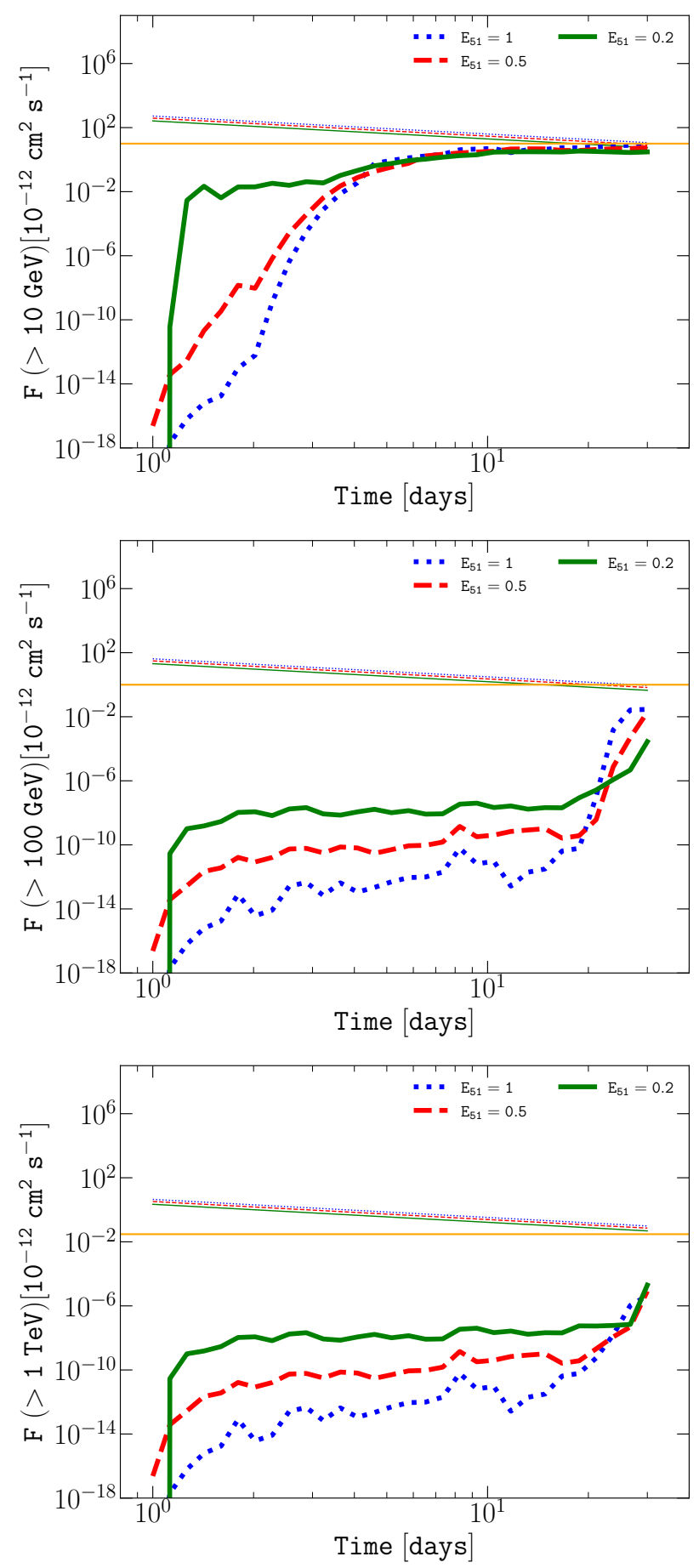

Figure 1: Time evolution of the integrated gamma-ray signal from a type IIP SN located at $1 \mathrm{Mpc}, M_{\mathrm{ej}}=10$ $\mathrm{M}_{\odot}, \dot{M}=10 \mathrm{M}_{\odot} / \mathrm{yr}$. The unattenuated signal is shown (thin lines) and the integrated signal above $>10 \mathrm{GeV}$, $>100 \mathrm{GeV}$ and $>1 \mathrm{TeV}$ are shown (from top to bottom), for a total explosion energy $E_{\mathrm{SN}}=0.2$ (green solid), 0.5 (red dashed) and 1 (blue dotted) $\times 10^{51} \mathrm{erg}$. The typical sensitivity of CTA (orange horizontal line) for 50 hours is shown as a guiding-eye for the reader [24], a 5 hours integration curve would be roughly shift by a factor upward. 


\section{Conclusion}

We have calculated the high energy gamma-ray signal expected from type IIp cc-SNe, the most common objects in this category. The calculation account for the effect of time-dependent anisotropic gamma-gamma absorption effect over the soft photon field produced by the supernova photosphere. We find that due to this effect type IIp SNe can hardly be detected above $100 \mathrm{GeV}$ beyond one Mpc but the flux between 1-10 GeV is largely unattenuated. However, enhanced mass loss by the SN progenitor star a few years before the explosion could allow to shift this limit. The dectability horizon of Cherenkov telescopes could be larger for other cc-SNe such as type IIb or IIn.

\section{References}

[1] V. Tatischeff, Radio emission and nonlinear diffusive shock acceleration of cosmic rays in the supernova SN 1993J, A\&A 499 (2009) 191 [0903.2944].

[2] A.R. Bell, K.M. Schure, B. Reville and G. Giacinti, Cosmic-ray acceleration and escape from supernova remnants, MNRAS 431 (2013) 415 [1301. 7264].

[3] K.M. Schure and A.R. Bell, From cosmic ray source to the Galactic pool, MNRAS 437 (2014) 2802 [1310.7027].

[4] A. Marcowith, M. Renaud, V. Dwarkadas and V. Tatischeff, Cosmic-ray acceleration and gamma-ray signals from radio supernova, Nuclear Physics B Proceedings Supplements 256 (2014) 94 [1409.3670].

[5] K. Murase, T.A. Thompson and E.O. Ofek, Probing cosmic ray ion acceleration with radio-submm and gamma-ray emission from interaction-powered supernovae, MNRAS 440 (2014) 2528 [1311.6778].

[6] M. Cardillo, E. Amato and P. Blasi, On the cosmic ray spectrum from type II Supernovae, arXiv e-prints (2015) arXiv:1507.06086 [1507.06086].

[7] G. Giacinti and A.R. Bell, Collisionless shocks and TeV neutrinos before Supernova shock breakout from an optically thick wind, MNRAS 449 (2015) 3693 [1503 . 04170].

[8] V.N. Zirakashvili and V.S. Ptuskin, Type IIn supernovae as sources of high energy astrophysical neutrinos, Astroparticle Physics 78 (2016) 28 [1510 . 08387].

[9] M. Petropoulou, S. Coenders, G. Vasilopoulos, A. Kamble and L. Sironi, Point-source and diffuse high-energy neutrino emission from Type IIn supernovae, MNRAS 470 (2017) 1881 [1705.06752].

[10] A.M. Bykov, D.C. Ellison, P.E. Gladilin and S.M. Osipov, Supernovae in compact star clusters as sources of high-energy cosmic rays and neutrinos, Advances in Space Research 62 (2018) 2764 [1706.01135].

[11] A.M. Bykov, D.C. Ellison, A. Marcowith and S.M. Osipov, Cosmic Ray Production in Supernovae, Space Sci. Rev. 214 (2018) 41 [1801.08890]. 
[12] A. Marcowith, V.V. Dwarkadas, M. Renaud, V. Tatischeff and G. Giacinti, Core-collapse supernovae as cosmic ray sources, MNRAS 479 (2018) 4470 [1806.09700].

[13] I. Tamborra and K. Murase, Neutrinos from Supernovae, Space Sci. Rev. 214 (2018) 31.

[14] K. Murase, A. Franckowiak, K. Maeda, R. Margutti and J.F. Beacom, High-energy Emission from Interacting Supernovae: New Constraints on Cosmic-Ray Acceleration in Dense Circumstellar Environments, ApJ 874 (2019) 80 [1807. 01460].

[15] K. Fang, B.D. Metzger, K. Murase, I. Bartos and K. Kotera, Multimessenger Implications of AT2018cow: High-energy Cosmic-Ray and Neutrino Emissions from Magnetar-powered Superluminous Transients, ApJ 878 (2019) 34 [1812 . 11673].

[16] P. Cristofari, M. Renaud, A. Marcowith, V.V. Dwarkadas, V. Tatischeff and G. Giacinti, Would SN1993J Have Been Detected by Next-generation Cerenkov Instruments?, Research Notes of the American Astronomical Society 4 (2020) 115.

[17] P. Cristofari, M. Renaud, A. Marcowith, V.V. Dwarkadas and V. Tatischeff, Time-dependent high-energy gamma-ray signal from accelerated particles in core-collapse supernovae: the case of SN 1993J, MNRAS 494 (2020) 2760 [2004.02650].

[18] Cherenkov Telescope Array Consortium, B.S. Acharya, I. Agudo, I. Al Samarai, R. Alfaro, J. Alfaro et al., Science with the Cherenkov Telescope Array (2019), 10.1142/10986.

[19] L.O. Drury, F.A. Aharonian and H.J. Voelk, The gamma-ray visibility of supernova remnants. A test of cosmic ray origin, A\&A 287 (1994) 959 [astro-ph/9305037].

[20] I. Rabinak and E. Waxman, The Early UV/Optical Emission from Core-collapse Supernovae, ApJ 728 (2011) 63 [1002.3414].

[21] X. Tang and R.A. Chevalier, Shock evolution in non-radiative supernova remnants, MNRAS 465 (2017) 3793 [1607.06391].

[22] S.J. Smartt, Progenitors of Core-Collapse Supernovae, ARA\&A 47 (2009) 63 [0908. 0700].

[23] P. Cristofari, P. Blasi and E. Amato, The low rate of Galactic pevatrons, Astroparticle Physics 123 (2020) 102492 [2007.04294].

[24] V. Fioretti, A. Bulgarelli and F. Schüssler, The Cherenkov Telescope array on-site integral sensitivity: observing the Crab, in Proceedings of the SPIE, Volume 9906, id. 9906308 pp. (2016)., vol. 9906 of Society of Photo-Optical Instrumentation Engineers (SPIE) Conference Series, p. 99063 O (2016), DOI. 